\title{
Regional surface morphology of comet 67P/Churyumov-Gerasimenko from Rosetta/OSIRIS images: The southern hemisphere (Corrigendum)
}

M. R. El-Maarry ${ }^{1}$, N. Thomas ${ }^{1}$, A. Gracia-Berná ${ }^{1}$, M. Pajola ${ }^{29}$, J.-C. Lee ${ }^{3}$, M. Massironi ${ }^{4}$, B. Davidsson ${ }^{5}$, S. Marchi ${ }^{6}$, H. U. Keller ${ }^{7}$, S. F. Hviid ${ }^{8}$, S. Besse ${ }^{9}$, H. Sierks ${ }^{10}$, C. Barbieri ${ }^{11}$, P. L. Lamy ${ }^{12}$, D. Koschny ${ }^{9}$, H. Rickman ${ }^{15,16}$, R. Rodrigo ${ }^{13,14}$, M. F. A'Hearn ${ }^{17}$, A.-T. Auger ${ }^{12}$, M. A. Barucci ${ }^{18}$, J.-L. Bertaux ${ }^{19}$, I. Bertini ${ }^{2}$, D. Bodewits ${ }^{17}$, G. Cremonese, ${ }^{11}$, V. Da Deppo ${ }^{20}$, M. De Cecco $^{21}$, S. Debei ${ }^{22}$, C. Güttler ${ }^{10}$, S. Fornasier ${ }^{19}$, M. Fulle ${ }^{23}$, L. Giacomini ${ }^{4}$, O. Groussin ${ }^{12}$, P. J. Gutierrez ${ }^{24}$, W.-H. Ip ${ }^{25}$, L. Jorda ${ }^{26}$, J. Knollenberg ${ }^{8}$, G. Kovacs ${ }^{10}$, J.-R. Kramm ${ }^{10}$, E. Kührt ${ }^{8}$, M. Küppers ${ }^{27}$, L. M. Lara ${ }^{24}$, M. Lazzarin ${ }^{11}$, J. J. Lopez Moreno ${ }^{24}$, R. Marschall ${ }^{1}$, F. Marzari ${ }^{11}$, G. Naletto ${ }^{2,20,28}$, N. Oklay ${ }^{8}$, A. Pommerol ${ }^{1}$, F. Preusker ${ }^{8}$, F. Scholten ${ }^{8}$, C. Tubiana ${ }^{10}$, and J.-B. Vincent ${ }^{8}$

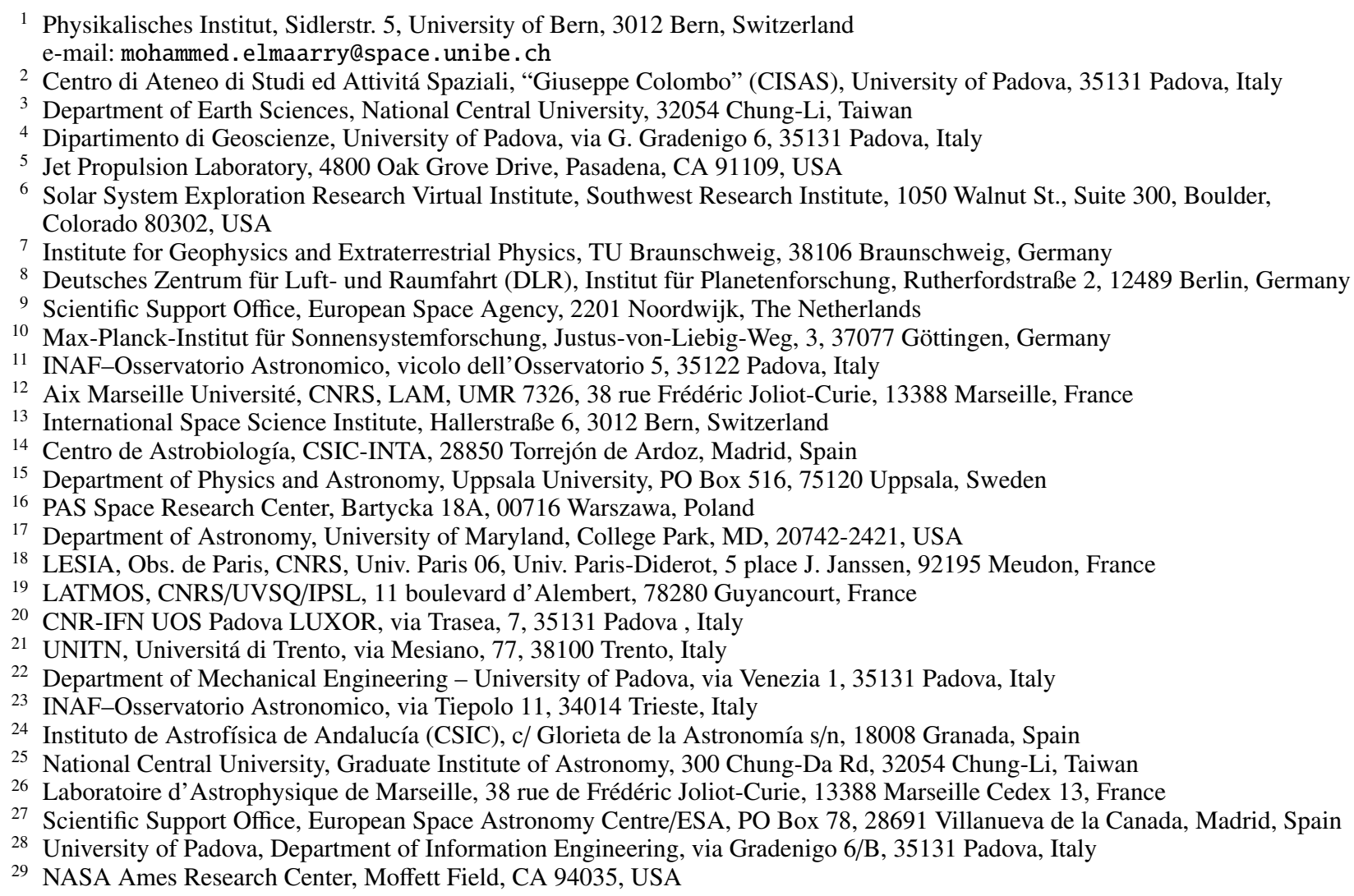

A\&A 593, A110 (2016), DOI: 10.1051/0004-6361/201628634

Key words. comets: general - comets: individual: 67P/Churyumov-Gerasimenko - methods: observational - errata, addenda

Following the paper's release, we realized there were some inconsistencies and/or typos in Figs. 1, 2, 6 and 9 regarding some of the regional boundaries and labels. Here we show the corrected figures. In Fig. 1, we modified the boundary between Anhur and Khepry in the middle panel and the boundary between Sobek and Anuket in the bottom panel. The latter was inconsistent with the correct boundary shown in Fig. 9. In Fig. 2, there were mistakes in the regional labels of the middle right panel with regards to Bes, Babi, and Anhur and have now been corrected. In Fig. 6, we modified the boundary between Anhur and Khepry corresponding to the same correction in Fig. 1. Finally, in Fig. 9, we modified the boundary between Maftet and Wosret to be consistent with the middle panel in Fig. 1.

Acknowledgements. The authors would like to thank Andrew Cooper and Marco Parigi for spotting the inconsistencies in the original figures following the release of the paper. 


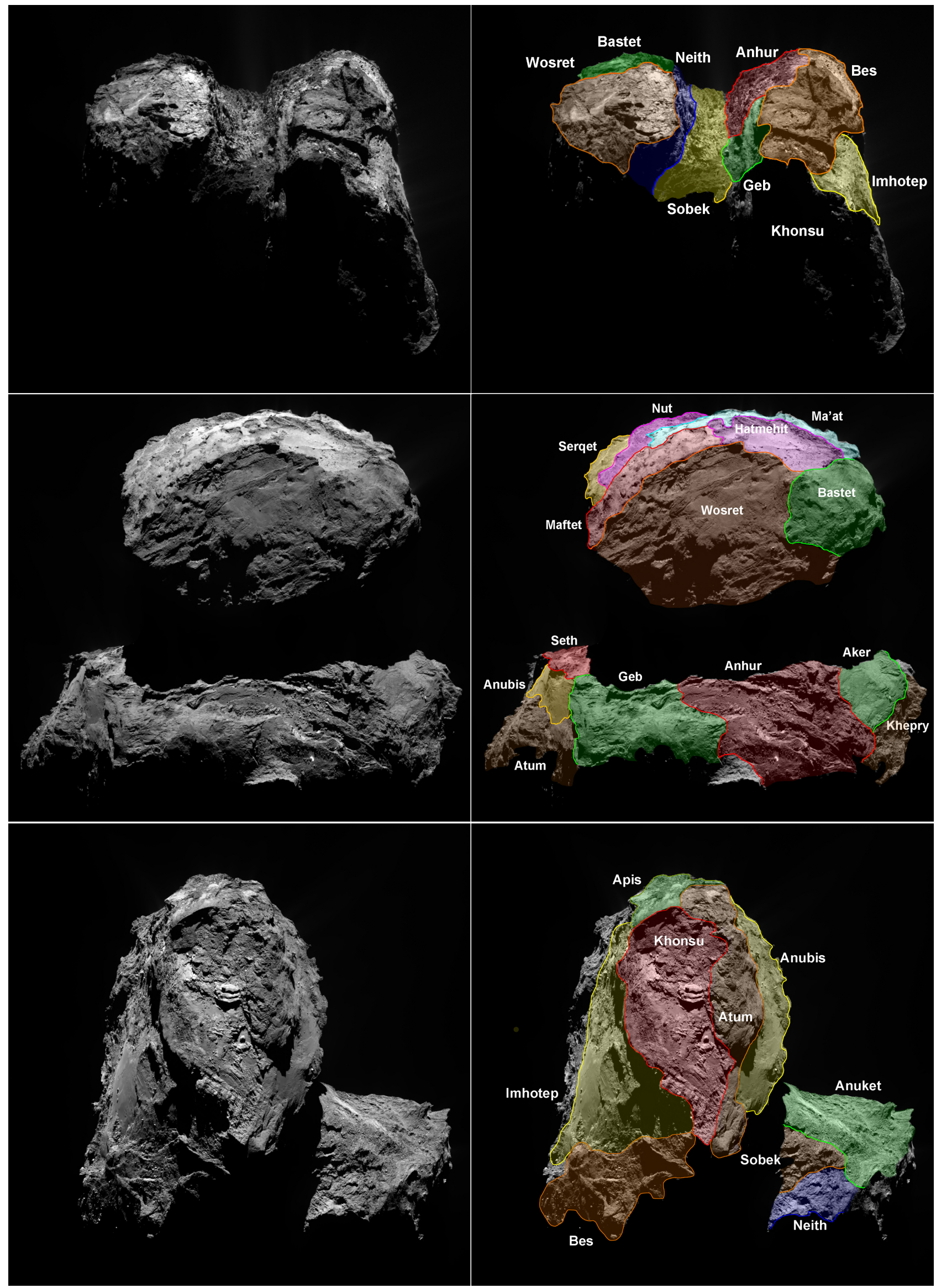

Fig. 1. Different views of the comet's southern hemisphere alongside similar orientations with regional boundaries overlaid. 


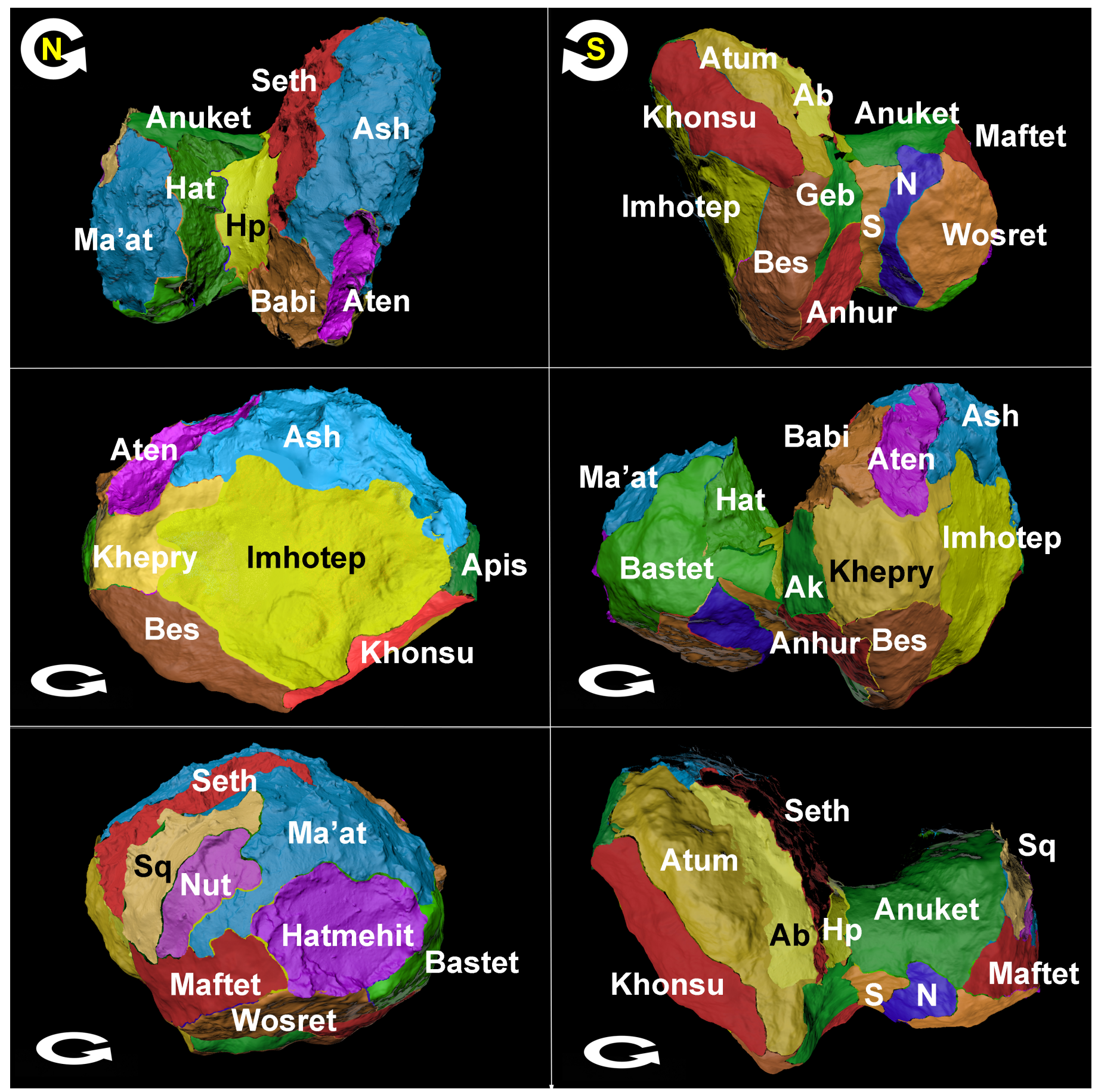

Fig. 2. Six different viewing angles showing all the defined regions, including the northern hemisphere, on a shape model of the comet for global context and orientation. Acronyms used correspond to Hapi (Hp), Hathor (Hat) Sobek (S), Neith (N), Aker (Ak), and Serqet (Sq). Circular arrows show the direction of the comet's rotation. The two upper panels display the comet from a northern and southern polar viewing angles. The remaining four panels show the comet from an equatorial projection each with a shift of $\sim 90$ degrees. 


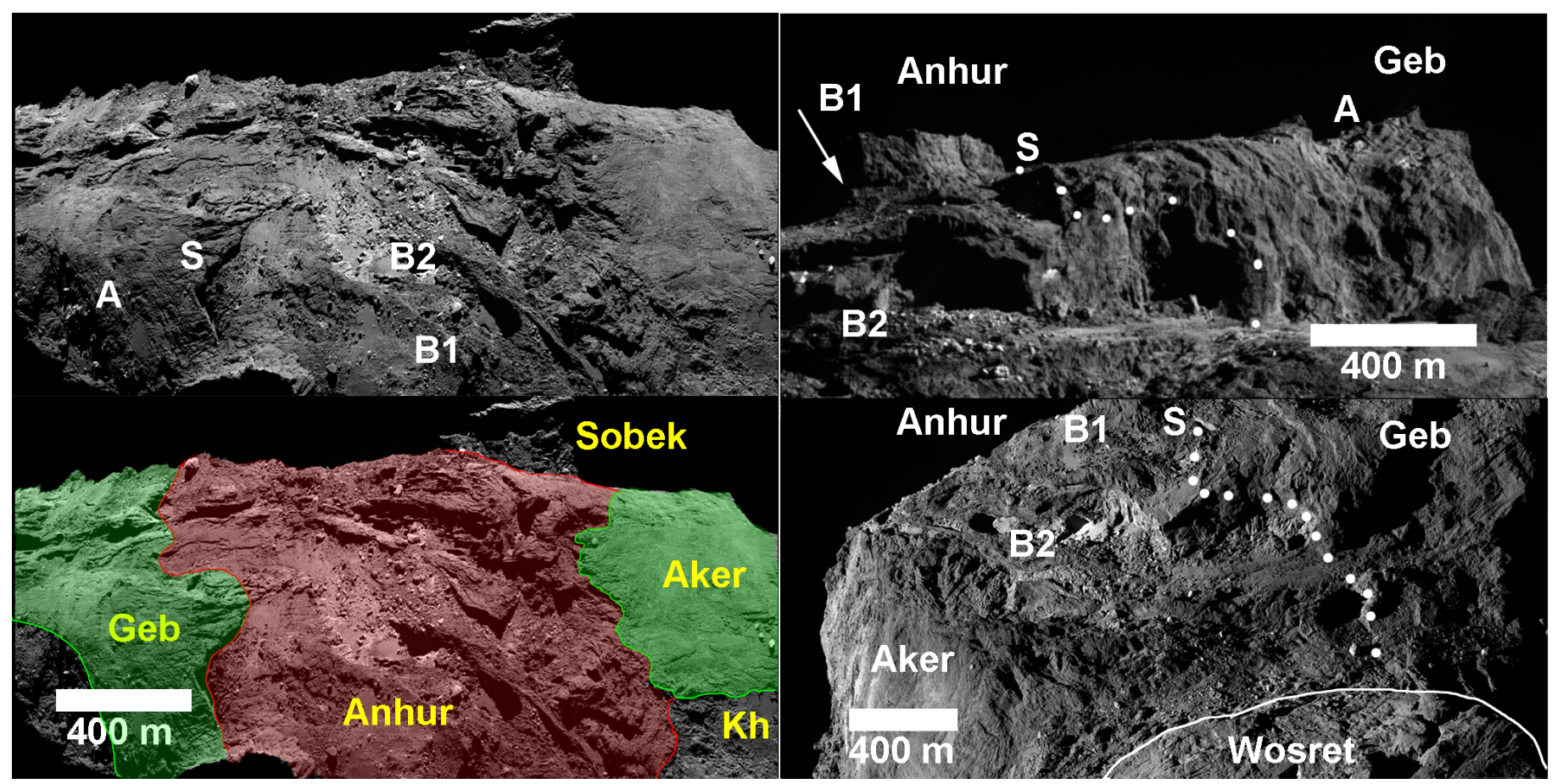

Fig. 6. Left: NAC image showing the main cliff of the large lobe's southern hemisphere from a "top view" along with another version including colored overlays of the defined regions Anhur and Geb and their boundaries. Geb appears to be more consolidated than Anhur as evident from the presence of various fracture patterns (A) and the lack of boulder fields (e.g., B1 and B2 in Anhur). The letter "S" shows the location of a welldefined scarp that separates the two cliff regions. The letters are representative of the same features marked in all panels. Upper right: a "head-on" view of the cliffs highlighting the morphological aspects that have already been mentioned, particularly of Geb. The dots show the location of the boundary marked clearly in the colored overlay of the left panel. Lower right: similar view of the cliffs highlighting the surface texture of Anhur. 


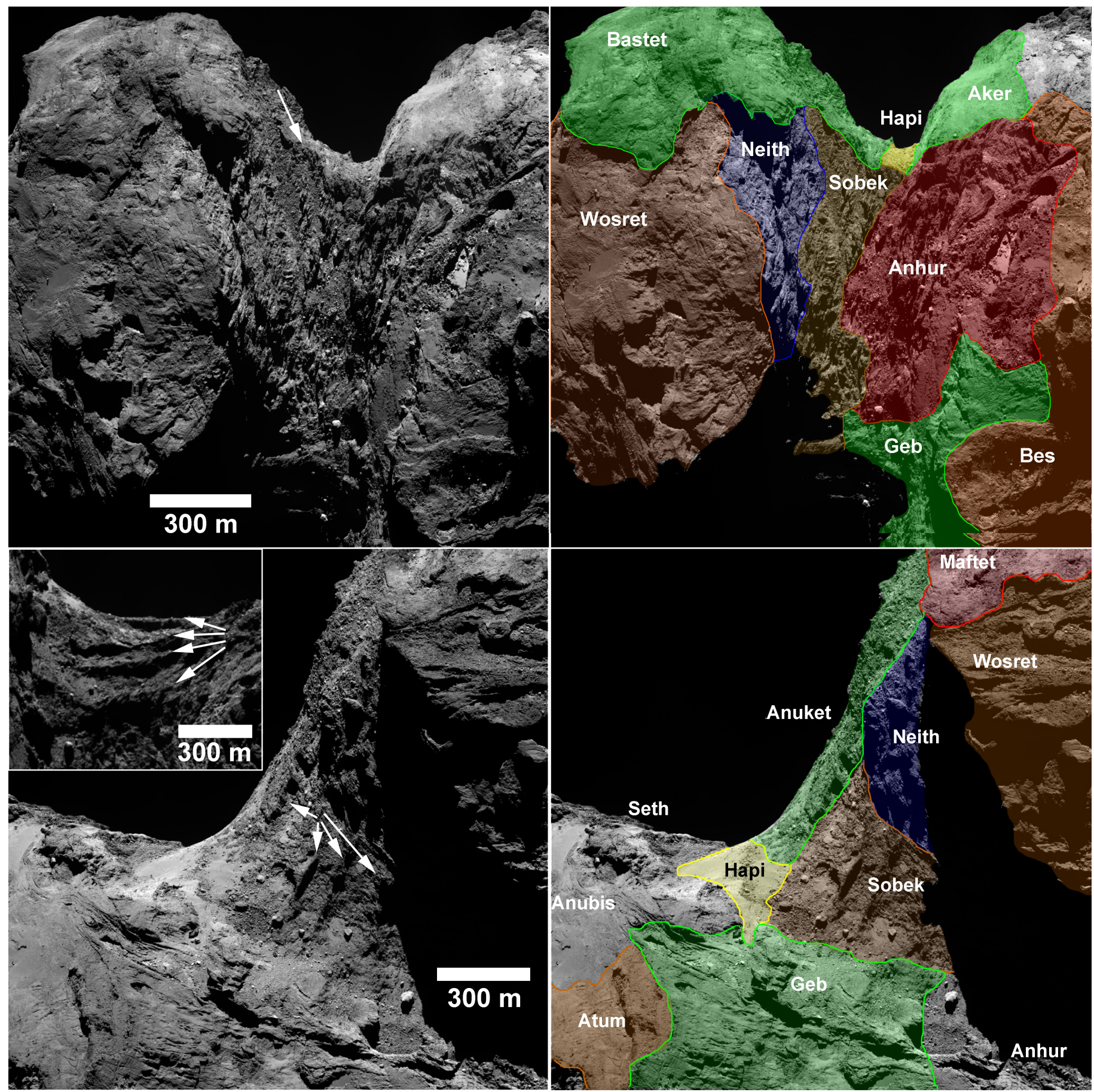

Fig. 9. Top: NAC image showing a "view from the top" of the southern hemisphere including a significant portion of the neck region Sobek along with a similar view including a colored overlay of the regions and their boundaries. Note the difficulty in differentiating Sobek from Neith on account of morphology. The arrow highlights the boundary with Bastet, which appears to overlie the neck region. Bottom: another view of the southern hemisphere with a similar color overlay highlighting the boundaries of Sobek with neighboring regions on the side opposite to that shown in the top panel. Note the arrows that show evidence for terraces starting from the contact with Anuket, which is better shown in the view taken at different geometry and illumination conditions (inbox). As the image in the box was taken from a longer distance (i.e., lower resolution), it has been resampled using a bicubic interpolation in order to show the terraces more clearly. 